Complexities in structure and function of the lung. New York: MarcelDekker; 1998. pp. 511-547.

8. Arai TJ, Henderson AC, Dubowitz DJ, Levin DL, Friedman PJ, Buxton RB, et al. Hypoxic pulmonary vasoconstriction does not contribute to pulmonary blood flow heterogeneity in normoxia in normal supine humans. J Appl Physiol (1985) 2009;106:1057-1064.

Copyright () 2018 by the American Thoracic Society

\section{Reply to Dorrington et al. and to Swenson}

From the Authors:

We want to thank Dr. Dorrington and colleagues and Dr. Swenson for their interest in our paper (1). We agree that hypercapnic pulmonary vasoconstriction is underappreciated as an independent component of the vascular response. However, our estimates suggest that $\mathrm{CO}_{2}$ fails as a singular explanation for the nonhypoxic component of perfusion redistribution during bronchoconstriction, and that other possible mechanisms, such as those we propose in the article, should be considered.

For a quantitative estimate of the contribution of hypercapnia to the decrease in regional perfusion during bronchoconstriction in our study, we rely on results from animal studies reporting relative reductions in regional perfusion per $\mathrm{mm} \mathrm{Hg}$ hypercapnia $(\% / \mathrm{mm} \mathrm{Hg})$ of 0.35 in cats (2), 0.97 (2) and 0.64 (3) in dogs, and 1.9 (4) in sheep. In addition, it is known that human subjects during mild bronchoconstriction do not develop hypercapnia (5-7) and would be expected to have an arteriovenous difference in $\mathrm{PCO}_{2}$ of $\sim 6 \mathrm{~mm} \mathrm{Hg}$ (8). Thus, assuming, based on the animal data, a reduction in perfusion of $\sim 1 \% / \mathrm{mm} \mathrm{Hg}$ in humans, the upper limit for hypercapnia with an increase of $6 \mathrm{~mm}$ $\mathrm{Hg}$ in severely constricted hypoventilated regions would explain a relative reduction in perfusion of only $6 \%$. For comparison with our study, we chose a representative data point of average values in our subregion analysis that included a narrow range of $\mathrm{V} / \mathrm{Q}$, a low $\mathrm{PO}_{2}$ breathing air, and a degree of hypoventilation allowing for regional $\mathrm{PO}_{2}$ greater than $100 \mathrm{~mm} \mathrm{Hg}$ breathing $100 \% \mathrm{O}_{2}$, but a $\mathrm{CO}_{2}$ as close as possible to the mixed venous level. A change in alveolar $\mathrm{PO}_{2}$ from $61 \mathrm{~mm} \mathrm{Hg}$ at room air to $291 \mathrm{~mm} \mathrm{Hg}$ at $100 \% \mathrm{O}_{2}$ resulted in a relative change in perfusion after methacholine treatment from $-31.2 \%$ to $-16.5 \%$, respectively. Thus, the estimated $6 \%$ reduction in perfusion in response to hypercapnia is indeed a relevant contribution but accounts for only a small fraction of the remaining nonhypoxic decrease of $-16.5 \%$ and falls well short as a singular explanation. Contributions of other factors to vasoconstriction are known for lobar collapse (9), which likely involves the loss of parenchymal tethering forces that counterbalance vascular smooth muscle constriction. In contrast, bronchoconstriction may affect the vascular tree by distorting vessels adjacent to the constricted airway or by stimulating vascular smooth muscle. Dr. Swenson suggests the use of a correlation analysis to determine the contribution of $\mathrm{PCO}_{2}$ to the perfusion redistribution, but the regional bronchoconstriction in ventilation defects would affect

Originally Published in Press as DOI: 10.1164/rccm.201709-1892LE on October 26, 2017 both $\mathrm{PCO}_{2}$ and any other component of the vascular response, resulting in correlated inputs.

In conclusion, hypercapnia in ventilation defects certainly contributes to vasoconstriction and changes in regional perfusion during bronchoconstriction, but the magnitude of the nonhypoxic reduction in regional blood flow compared with the estimated effect of hypercapnia is many-fold higher. This suggests that other mechanisms, such as parenchymal forces or a vascular smooth muscle agonist, have a substantial effect on the regional change in vascular resistance during bronchoconstriction.

Author disclosures are available with the text of this letter at www.atsjournals.org.

Vanessa J. Kelly, Ph.D.

Tilo Winkler, Ph.D.

R. Scott Harris, M.D.

Harvard Medical School

Boston, Massachusetts

On behalf of all authors

\section{References}

1. Kelly VJ, Hibbert KA, Kohli P, Kone M, Greenblatt EE, Venegas JG, et al. Hypoxic pulmonary vasoconstriction does not explain all regional perfusion redistribution in asthma. Am J Respir Crit Care Med 2017; 196:834-844.

2. Barer GR, Howard P, Shaw JW. Stimulus-response curves for the pulmonary vascular bed to hypoxia and hypercapnia. J Physiol 1970; 211:139-155.

3. Noble WH, Kay JC, Fisher JA. The effect of PCO2 on hypoxic pulmonary vasoconstriction. Can Anaesth Soc J 1981;28:422-430.

4. Sheehan DW, Farhi LE. Local pulmonary blood flow: control and gas exchange. Respir Physiol 1993;94:91-107.

5. McFadden ER Jr, Lyons HA. Arterial-blood gas tension in asthma. N Engl J Med 1968;278:1027-1032.

6. Miyamoto T, Mizuno K, Furuya K. Arterial blood gases in bronchial asthma. J Allergy 1970;45:248-254.

7. Echazarreta AL, Gómez FP, Ribas J, Sala E, Barberà JA, Roca J, et al. Pulmonary gas exchange responses to histamine and methacholine challenges in mild asthma. Eur Respir J 2001;17:609-614.

8. Adrogué HJ, Rashad MN, Gorin AB, Yacoub J, Madias NE. Assessing acidbase status in circulatory failure. Differences between arterial and central venous blood. N Engl J Med 1989;320:1312-1316.

9. McFarlane PA, Gardaz JP, Sykes MK. CO2 and mechanical factors reduce blood flow in a collapsed lung lobe. J Appl Physiol 1984;57:739-743.

Copyright $@ 2018$ by the American Thoracic Society

\section{Erratum: Fifty Years of Research in ARDS. Setting Positive End-Expiratory Pressure in Acute Respiratory Distress Syndrome}

There is an error in the article by Sahetya and colleagues (1), published in the June 1, 2017, issue of the Journal. On page 1433, one of the sentences in the second paragraph under the heading DRIVING PRESSURE should read: "With constant tidal volume, if PEEP is raised and driving pressure decreases, the $\mathrm{C}_{\mathrm{RS}}$ has increased, suggesting that the higher PEEP caused lung recruitment." The published version of the sentence incorrectly stated that the $C_{R S}$ would have decreased. The authors apologize for the error. 


\section{Reference}

1. Sahetya SK, Goligher EC, Brower RG. Fifty years of research in ARDS. Setting positive end-expiratory pressure in acute respiratory distress syndrome. Am J Respir Crit Care Med 2017;195:1429-1438.

Copyright (c) 2018 by the American Thoracic Society

\section{Erratum: Palivizumab Prophylaxis in Preterm Infants and Subsequent Recurrent Wheezing. Six-Year Follow-up Study}

The article by Mochizuki and colleagues (1) published in the July 1, 2017 , issue of the Journal contains errors in Table 3. The table is reprinted below with the corrected values appearing in bold. These errors do not affect the results or statistical findings of the paper. The authors apologize to the readership of the Journal for any inconvenience.

\section{Reference}

1. Mochizuki H, Kusuda S, Okada K, Yoshihara S, Furuya H, Simões EAF; Scientific Committee for Elucidation of Infantile Asthma. Palivizumab prophylaxis in preterm infants and subsequent recurrent wheezing: six-year follow-up study. Am J Respir Crit Care Med 2017;196:29-38.

Copyright () 2018 by the American Thoracic Society

Table 3. Multivariate Comparison of Outcomes in the Study Populations

\begin{tabular}{|c|c|c|c|c|}
\hline & Adjusted OR $(95 \% \mathrm{Cl})$ & $P$ Value & Adjusted HR (95\% Cl) & $P$ Value \\
\hline \multicolumn{5}{|l|}{ Intention-to-treat population } \\
\hline Recurrent wheezing, $n=440$ & $0.34(0.19-0.60)$ & $<0.001$ & $0.38(0.23-0.62)$ & $<0.001$ \\
\hline Recurrent wheezing: family history of allergy, $n=250$ & $0.48(0.26-0.90)$ & 0.023 & $0.52(0.31-0.87)$ & 0.012 \\
\hline Recurrent wheezing: no family history of allergy, $n=190$ & $0.55(0.20-1.55)$ & 0.26 & $0.59(0.23-1.53)$ & 0.28 \\
\hline \multicolumn{5}{|l|}{ Per-protocol population } \\
\hline Recurrent wheezing, $n=328$ & $0.36(0.20-0.67)$ & 0.001 & $0.38(0.23-0.65)$ & $<00.001$ \\
\hline Recurrent wheezing: family history of allergy, $n=200$ & $0.28(0.13-0.60)$ & 0.001 & $0.31(0.16-0.60)$ & $<00.001$ \\
\hline Recurrent wheezing: no family history of allergy, $n=128$ & $0.55(0.18-1.63)$ & 0.278 & $0.52(0.20-1.37)$ & 0.187 \\
\hline \multicolumn{5}{|l|}{ Atopic asthma population } \\
\hline Recurrent wheezing, $n=268$ & $0.43(0.22-0.84)$ & 0.013 & $0.46(0.26-0.81)$ & 0.007 \\
\hline Recurrent wheezing: family history of allergy, $n=171$ & $0.26(0.12-0.58)$ & 0.001 & $0.30(0.16-0.59)$ & $<00.001$ \\
\hline Recurrent wheezing: no family history of allergy, $n=97$ & $1.86(0.38-9.25)$ & 0.45 & $1.81(0.40-8.12)$ & 0.44 \\
\hline Atopic asthma, $n=268$ & $0.79(0.37-1.69)$ & 0.54 & N/A & \\
\hline Atopic asthma: family history of allergy, $n=171$ & $0.68(0.13-3.47)$ & 0.24 & $\mathrm{~N} / \mathrm{A}$ & \\
\hline Atopic asthma: no family history of allergy, $n=97$ & $1.71(0.70-4.18)$ & 0.64 & $\mathrm{~N} / \mathrm{A}$ & \\
\hline
\end{tabular}

Definition of abbreviations: $\mathrm{Cl}=$ confidence interval; $\mathrm{HR}=$ hazard ratio; $\mathrm{N} / \mathrm{A}=$ not applicable: $\mathrm{HR}$ could not be calculated because the assessment was made only at $6 \mathrm{yr}$ of age; $\mathrm{OR}=$ odds ratio.

Treatment effects adjusted for gestational age, smokers in the home, and a family history of allergy. 